\title{
El Teletrabajo en el Ecuador
}

\author{
Teleworking in Ecuador
}

Diana Maricela Bermúdez Santana. ${ }^{1}$ \& Alberto Mauricio Pangol Lascano. ${ }^{2}$

DOI: https://doi.org/10.33262/visionariodigital.v5i4.1875

\begin{abstract}
.
Teleworking is a phenomenon that has become globalized and is emerging with greater force due to the pandemic caused by COVID-19; brings with it the use of technologies and an almost total immersion in the digital world, so that the current needs of work have bordered unimaginable levels of development of information and communication technologies, considered today as new forms of organization of the work and as primary tools to perform functions entrusted under a dependency relationship, which do not merit the presence of the worker, but are carried out in the home or another place outside the usual one. Objectives: Its objective is to determine the scope of legal protection that prevents or avoids long periods of digital connection, generating psychosocial risks that affect the physical, mental or emotional health of workers. Teleworking could violate
\end{abstract}

\footnotetext{
${ }^{1}$ Abogada, Magister en Derecho Penal y Procesal Penal por la Universidad Diego Portales. Abogada en libre ejercicio, actualmente, Docente en la Facultad de Jurisprudencia, Ciencias Políticas y Económicas, carrera de Derecho de la Universidad Tecnológica Indoamérica, sede Ambato, Ecuador. Correo electrónico dianabermudez@uti.edu.ec, dbermudezsantana@gmail.com, ORCID: https://orcid.org/0000-0003-3220-0990.

${ }^{2}$ Licenciado en Ciencias Políticas y Sociales, Abogado de los Juzgados y Tribunales de la República del Ecuador, Doctor en Jurisprudencia, Diploma Superior en Contratación Laboral, Especialista en Consultoría Jurídica Laboral, Magister en Derecho Laboral, Diploma Superior en Derecho Procesal, Especialista en Derecho Procesal, Magister en Derecho Procesal con mención en Derecho Civil, Candidato a Doctor en Ciencias Jurídicas por la Pontificia Universidad Católica Argentina, Decano y profesor a tiempo completo de la Facultad de Jurisprudencia, Ciencias Políticas y Económicas, de la Universidad Tecnológica Indoamérica, UTI, Abogado en libre ejercicio, Asesor de la Comisión Especializada de los Trabajadores y Seguridad Social, Juez Temporal de los Juzgados del Trabajo, Delegado del Consejo de la Judicatura de la provincia de Tungurahua, Referente Nacional por el Ecuador de la Sección Latinoamericana de los Jóvenes Juristas de la Sociedad Internacional de Derecho del Trabajo y de la Seguridad Social (SIDTSS), Miembro activo de la Sociedad Ecuatoriana de Derecho del Trabajo y de la Seguridad Social (SEDTSS), Delegado Principal a la Asamblea Nacional de la Federación de Abogados, Representante del Colegio de Abogados de Tungurahua, Referente Nacional por el Ecuador de la Sección Latinoamericana de los Jóvenes Juristas de la Sociedad Internacional de Derecho del Trabajo y de la Seguridad Social (SIDTSS), https://orcid.org/0000-0002-5093-4165, maurop7@hotmail.com, albertopangol@uti.edu.ec.
} 
human rights such as digital disconnection, time off and enjoying vacations. Methodology: For this, the historical-descriptive research method was used, of a documentary type of bibliographic review, in which the internal and international legislation on normative reforms regarding teleworking and the right to disconnection as fundamental axes in labor matters is described. Contributions: Telework generates a number of positive and negative contributions to society, therefore, it is important that the current legal regulations related to the subject be analyzed from a descriptive point of view that generates knowledge to workers, companies and about everything is avoided the repercussions of an inadequate treatment to this type of work.

Keywords: law, telework, Tics, work, right to disconnect

\section{Resumen.}

El Teletrabajo es un fenómeno que se ha globalizado y surge con mayor fuerza a causa de la pandemia provocada por el COVID-19; trae consigo el uso de tecnologías y una inmersión casi total en el mundo digital, por lo que las necesidades actuales del trabajo han bordeado niveles inimaginables de desarrollo de las tecnologías de la información y la comunicación, consideradas hoy en día como nuevas formas de organización del trabajo y como herramientas primordiales para desempeñar funciones encomendadas bajo relación de dependencia, las cuales no ameritan la presencia del trabajador, sino que, se desarrollan en el domicilio u otro lugar ajeno al habitual. Objetivos: Tiene como objetivo, determinar el alcance de la protección jurídica que previene o evita períodos extensos de conexión digital, generando riesgos de tipo psicosocial que afectan la salud física, mental o emocional de los trabajadores. El teletrabajo podría vulnerar derechos humanos como la desconexión digital, tiempo de descanso y goce de vacaciones. Metodología: Para ello, se utilizó el método de investigación histórico-descriptivo, de tipo documental de revisión bibliográfica, en la cual se describe la legislación interna e internacional sobre reformas normativas referentes al teletrabajo y derecho a la desconexión como ejes fundamentales en materia laboral. Aportes: El Teletrabajo genera un sin numero de aportes positivos y negativos a la sociedad, por lo tanto, es importante que la normativa legal vigente relacionada al tema, sea analizada desde un punto de vista descriptivo que genere conocimientos a los trabajadores, empleadores y sobre todo se evite las repercusiones de un trato inadecuado a esta modalidad de trabajo.

Palabras claves: Derecho, teletrabajo, Tics, trabajo, derecho a la desconexión.

\section{Introducción.}

La realidad actual que atraviesa el Ecuador y el mundo a causa de la pandemia generada por el Covid-19, ha ocasionado que las modalidades de trabajo tengan que mutar y adecuarse a una nueva normalidad, llevada a cabo bajo algunos estándares en los cuales los Estados tomaban el protagonismo. Cada gobierno emitió medidas obligatorias de 
confinamiento, con la finalidad de frenar la expansión del virus, sin embargo, implicó también que los trabajadores se transformen en teletrabajadores, sin la necesidad de que exista una autorización por parte del empleador; la finalidad era no paralizar de forma total las actividades laborales. Esto implicó que el proceso del trabajo mediante el uso de las Tecnologías de la Información y la Comunicación (TIC), sea acelerado, y que el teletrabajo se posesione como la modalidad que permitía al trabajador continuar cumpliendo funciones de carácter laboral, pero no desde las instalaciones que imponga el empleador, sino desde sus domicilios o desde un lugar externo, fuera del establecido o fijado por parte del empleador.

En los últimos años el trabajo sufrió una modificación considerable, al desarrollar trabajo por medio de dispositivos tecnológicos que permiten la prestación de servicios lícitos y personales, pero sin requerir la presencialidad, como usualmente se solicita en los trabajos. El teletrabajo como una nueva forma de trabajar, cimienta su desarrollo en la obtención de resultados, y un desenvolvimiento flexible y autónomo, el cual al encontrarse implementado en la cotidianeidad requiere que la norma brinde una protección de derechos, los cuales se relacionan con el teletrabajo y al mismo tiempo con una garantía que permita su efectivo cumplimiento.

El teletrabajo es una forma de realizar el trabajo mediante el uso de las TIC, pero esto no elimina la relación de dependencia con el empleador, generándose una interrogante respecto a las funciones de dirección o administración que cumple el empleador o la persona encargada, sin embargo, la legislación tanto nacional como internacional, establecen directrices que permitan sobrellevar este tipo de modalidad de trabajo en base a los estándares establecidos que se fundan en la comunicación y el cumplimiento de metas o productos asignados, independientemente de las horas de trabajo que dedique al cumplimiento de las labores encomendadas. Además, en cuanto a las consideraciones al empleador, el trabajar de forma telemática, reduce los costos generados en el lugar de trabajo, pero, cabe mencionar la responsabilidad del empleador de dotar las herramientas necesarias para el desarrollo de las actividades delegadas.

Uno de los derechos relacionados con el teletrabajo y la digitalización es el derecho a la desconexión digital, considerada ésta como un elemento esencial que precautela la salud de los teletrabajadores; y que al mismo tiempo garantiza el respeto al tiempo de descanso y a las vacaciones de la cual gozan los trabajadores; en este derecho está incluido el respeto a la intimidad de tipo personal y familiar. El período de descanso de los teletrabajadores es cada vez un tema de importancia, dados los procesos de revolución digital que atraviesa el mundo entero. La brecha que podría existir entre el trabajo y el descanso se está desvaneciendo, afectando de manera notable en el aspecto psicosocial, emocional e incluso físico de las personas.

Los efectos que genera el teletrabajo se reflejan en un ámbito no solo laboral, sino familiar e interpersonal, radicando ahí la importancia de realizar estudios y enfocar el análisis no solo en el punto de vista de la productividad dirigida hacia el empleador y la economía del país, sino desde el punto de vista de la salud y riesgos que podría provocar el 
desconocimiento de la modalidad de teletrabajo, y de llevarla a cabo de la manera correcta. Si bien es cierto, no genera una supervisión por parte del empleador, pero en la mayor cantidad de casos, quienes desarrollan la modalidad de teletrabajo dedican un número mayor de horas a sus labores, dejando sin importancia sus tiempos de desconexión.

Otro de los problemas que se genera debido a la modalidad del teletrabajo, es el hecho de que no existe una diferencia espacial entre el lugar de descanso y el lugar de trabajo, generando así que las personas se sientan agobiadas y cansadas de su entorno; a su vez hay que considerar que no solo una persona se encuentra trabajando desde casa, en muchos hogares los jefes de familia, tanto padre como madre, tienen jornadas laborales de manera simultánea, provocando un sinnúmero de incomodidades, las cuales disminuyen la productividad.

\section{Metodologia.}

Los resultados obtenidos provienen de la aplicación del método histórico - descriptivo, de tipo documental el cual deriva de una revisión bibliográfica, la cual consiste en la exploración de textos, libros, revistas, etc. que permitan sustentar los aportes expuestos, considerando los principales, aquellos que han sido planteados dentro de esta investigación; se ha realizado un análisis documental, en el cual de describen, clasifican y resumen las normativas referentes al teletrabajo, de carácter nacional e internacional; de igual manera se analizan las reformas normativas que se han realizado referentes al tema planteado; y todos los derechos conexos al Teletrabajo como una modalidad de trabajo actual.

\section{Resultados.}

La normativa del Ecuador, así como los Tratados y Convenios Internacionales tienen en consideración que existe incidencia de varios riesgos en la salud mental de las personas producto del teletrabajo en temporada de confinamiento, y que esto se ha convertido en un factor de riesgo. Uno de los fatores corresponde al estrés y los síntomas fisiológicos que esta causa, sumándose un sin número de circunstancias generadas por la pandemia del COVID-19, como el miedo al contagio y/o morir no solo personal sino familiar, el sedentarismo, sentimientos de impotencia, el temor en el área financiera por pérdida del empleo, rebaja de sueldos o inestabilidad laboral, así como las restricciones de movilidad y aislamiento del contexto social y familiar que se transforma en síntomas de ansiedad y depresión.

Es así como, se reconoce que la salud mental es primordial, pues de eso se trata la raíz de lo que nos hace humanos; nuestros caminos, las personas con las que interactuamos, estudiamos, trabajamos, luchamos y conectamos, todo ello engloba una buena salud mental, lo cual facilita a las personas el desempeño de sus funciones en cualquier ámbito.

Es importante recordar que el impacto de la pandemia y sus riesgos por medio del teletrabajo, es una manera viable de accesibilidad al trabajo y a la generación de recursos, 
pero no es menos importante que la salud en general se encuentra inestable, pues los teletrabajadores a causa de esto también llegan a desarrollar un crecimiento del deterioro en la productividad en su área de trabajo y el crecimiento de ausentismo por un colapso y fatiga tanto corporal como mental.

Es por ello que, no es suficiente que la empresa o institución descanse a sus empleados en una desconexión digital, sino que, a su vez, se debería asumir roles de supervisión y control, pues la desconexión no debe interrumpirse. En este sentido, modifica las condiciones de vida, los representantes de los trabajadores deben promover políticas nacionales para los empleados, incluidos los que ocupan puestos de responsabilidad en cargos altos como el gerente, decidiendo cómo ejercer el derecho a terminar una jornada laboral con programas de formación y sensibilización sobre los medios digitales y de cómo utilizarlos correctamente con herramientas técnicas para evitar el riesgo de una salud mental y física en decadencia.

Finalmente se evidencia que el teletrabajo ha sido una salida para que las personas no pierdan sus trabajos, pero los más beneficiados por esto han sido las empresas, ya que al no utilizar sus instalaciones, han reducido sus costos, los servicios básicos, como luz, agua, y teléfono han pasado a ser parte de los gastos de los empleados; de igual forma, al no usar las instalaciones de la empresa, muchas de ellas han prescindido del personal de limpieza y mantenimiento, considerando que por obvias razones este tipo de trabajo, no se lo puede realizar de forma remota.

\section{Conclusiones.}

- La modalidad de teletrabajo tiene ventajas y desventajas desde diversos puntos de vista, una de las ventajas consideradas de trasladar el lugar de trabajo habitual al domicilio, provoca una reducción significativa de contaminación que podría mejorar las condiciones medioambientales; colaborar con la naturaleza; así mismo, permite generar una inclusión mucho más amplia en los ámbitos de trabajo para todas las personas, incluidas en estas personas con discapacidad, mujeres embarazadas, con enfermedades catastróficas, etc.

- El Teletrabajo permite flexibilizar las condiciones laborales, al realizar un trabajo desde el domicilio o lugar ajeno al habitual del trabajador, pero, es necesario precautelar que los trabajadores no incurran en gastos a causa de esta modalidad de trabajo, esto presupone entonces que, quien debe dotar de las herramientas necesarias para que el trabajador desarrolle sus actividades, es el empleador; teniendo en consideración que poseer un computador y acceso a internet, son los elementos esenciales.

- El tiempo libre, la recreación y el esparcimiento como derechos fundamentales, buscan evitar el riesgo mental que tienen los teletrabajadores; ya que el exceso de trabajo provocado por una falta de organización y respeto, por parte del empleador, de las horas de descanso, ocasionan una fatiga y frustración que puede verse reflejada en la convivencia con su entorno, esto quiere decir que su ambiente familiar puede deteriorarse debido a la demasía de trabajo. 
- Por lo tanto, dentro de la relación laboral, el empleador es el encargado de la protección en cuanto a la salud y seguridad de los trabajadores, incluidos en estos, aquellos que se desenvuelven como teletrabajadores, teniendo en consideración que independientemente de la modalidad, la responsabilidad frente a los riesgos de trabajo existe.

\section{Referencias bibliográficas.}

Aranda, J. (2002). Aspectos jurídicos del Teletrabajo. Revista del Ministerio de Trabajo y Asuntos Sociales(11), 44 . Obtenido de https://dialnet.unirioja.es/servlet/autor?codigo $=94651$

Asamblea Constituyente. (2008). Constitución de la República del Ecuador. Montecristi: Registro Oficial 449.

Beltrán, P. \& Sánchez, L. (2002). Las relaciones laborales virtuales: El Teletrabajo. Pontificia Universidad Javeriana.

Congreso Nacional. (s.f.). Código de Trabajo. Quito: Registro Oficial.

Eurofound. (2020). COVID-19 could permanently change teleworking in Europe. Dublin: Eurofound. 26 de marzo. Disponible en https://www.eurofound.europa.eu/news/newsarticles/covid-19-couldpermanently-change-teleworking-in-europe

Gómez, J. \& Moya R. (2020). La pandemia actual del COVID-19 en África. 6 de abril. Disponible en https://atalayar.com/content/la-pandemia-actual-del-covid-19-en$\% \mathrm{C} 3 \%$ A1frica

Lubiza, O. (2010). “El teletrabajo: una opción en la era digital”, Observatorio Laboral. Revista Venezolana, 3, N. ${ }^{\circ}$ 5. https://bit.ly/3eQarS0

Mejía, M. (2007). El teletrabajo en el mundo y Colombia. Gaceta Laboral. Obtenido de http://ve.scielo.org/scielo.php?pid=S1315$85972007000100002 \&$ script=sci_arttext\&tlng=pt

Ministerio de Trabajo Ecuador. (2016). Acuerdo No. MDT-2016-0190. Segundo Suplemento del Registro Oficial No.825. Obtenido de http://www.cit.org.ec/files/Resolucion-No.-3---MINTRA.pdf

Nieto, L. (2020). El teletrabajo de actor secundario a protagonista en el escenario del COVID-19. Diario La Ley. Obtenido de https://dialnet.unirioja.es/servlet/articulo?codigo=7589365

Organización Internacional del Trabajo [OIT]. (1919). Convenio $N^{o} 1$ sobre las horas de trabajo. ilo.org. Obtenido de ilo.org: https://www.ilo.org/dyn/normlex/es/f?p=NORMLEXPUB:12100:0::NO::P1210 0_ILO_CODE:C001 
Organización Internacional del Trabajo [OIT]. (1921). Convenio $N^{o} 14$ sobre el descanso semanal. ilo.org. Obtenido de ilo.org: https://www.ilo.org/dyn/normlex/es/f?p=NORMLEXPUB:12100:0::NO::P1210 0_ILO_CODE:C014

Organización Internacional del Trabajo [OIT]. (1935). Convenio $N^{o} 47$ sobre las cuarenta horas. ilo.org. Obtenido de ilo.org: https://www.ilo.org/dyn/normlex/es/f?p=NORMLEXPUB:12100:0::NO::P1210 0_ILO_CODE:C047

Organización Internacional del Trabajo [OIT]. (1957). Convenio $N^{o} 16$ sobre el descanso semanal. ilo.org. Obtenido de ilo.org: https://www.ilo.org/dyn/normlex/es/f?p=NORMLEXPUB:12100:0::NO::P1210 0_ILO_CODE:C106

Organización Internacional del Trabajo [OIT]. (1996). Convenio $N^{o} 177$ sobre el trabajo a domicilio. ilo.org. Obtenido de ilo.org: https://www.ilo.org/dyn/normlex/es/f?p=NORMLEXPUB:12100:0::NO::P1210 0_INSTRUMENT_ID:312322

Organización Internacional del Trabajo. (2017). ilo.org. Obtenido de ilo.org: https://www.ilo.org/dyn/normlex/es/f?p=1000:11200:0::NO:11200:P11200_CO UNTRY_ID:102616

Organización Internacional del Trabajo [OIT]. (2019). Digitalization and Decent Work: Implications for Pacific Island Countries. Suva, Oficina de País de la OIT para los Países Insulares del Pacífico. Disponible en https://www.ilo.org/wcmsp5/groups/public/---asia/---ro-bangkok/---ilosuva/documents/publication/wcms_712544.pdf

Organización Internacional del Trabajo [OIT]. (2020). El teletrabajo durante la pandemia de COVID-19 y después de ella. Primera edición. ilo.org. Obtenido de ilo.org: https://www.ilo.org/wcmsp5/groups/public/---ed_protect/---protrav/--travail/documents/pperisication/wcms_758007.pdf

Pardo, M., \& Rodríguez, M. (2020). El teletrabajo en tiempos de COVID-19. Universidad Católica de Colombia. Obtenido de https://hdl.handle.net/10983/24640

Peiró, J., \& Soler, A. (2020). El impulso al teletrabajo durante el COVID-19 y los retos que plantea. IvieLaB. Obtenido de https://umivale.es/dam/webcorporativa/Documentos-prevenci-n-y-salud/11.Covid19IvieExpress.Elimpulso-al-teletrabajo-durante-el-COVID-19-y-los-retos-que-planteaf.pdf

Sánchez, R. (2012). El teletrabajo como tendencia del mercado laboral. Retos: Revista de Ciencias de la Administración y Economía. Obtenido de https://reunir.unir.net/handle/123456789/5970 
Tomei, M. (1999). El trabajo a domicilio en los países seleccionados de América Latina: una visión comparativa, Documento de discusión, Oficina Internacional del Trabajo, Ginebra. ilo.org. Obtenido de ilo.org: https://www.ilo.org/wcmsp5/groups/public/---

ed_emp/documents/publication/wcms_123592.pdf

Ushakova, T. (2014). La cooperación de las organizaciones internacionales para la promoción de los estándares laborales en el contexto de la crisis: el papel de la OIT, Información Laboral, n. 4.

Visconti, E. (2019). El derecho a la desconexión digital en el ámbito laboral. Revista Vasca de Gestión de Personas y Organizaciones Públicas. Obtenido de https://www.ivap.euskadi.eus/contenidos/informacion/17_revgp/eu_def/Talens_ 150_161.pdf

Vittorio, D. (2004). El teletrabajo en América Latina y el Caribe. (Ginebra: Centro Internacional de Investigaciones para el Desarrollo). https://bit.ly/2BqgBtF

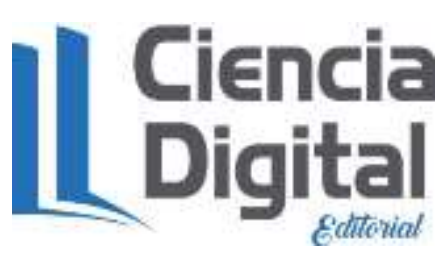




\section{PARA CITAR EL ARTÍCULO INDEXADO.}

Bermúdez Santana, D. M., \& Pangol Lascano, A. M. (2021). El Teletrabajo en el Ecuador . Visionario Digital, 5(4), 21-29. https://doi.org/10.33262/visionariodigital.v5i4.1875

\section{Liencia}

El artículo que se publica es de exclusiva responsabilidad de los autores y no necesariamente reflejan el pensamiento de la Revista Visionario Digital.

El artículo queda en propiedad de la revista y, por tanto, su publicación parcial y/o total en otro medio tiene que ser autorizado por el director de la Revista Visionario Digital.
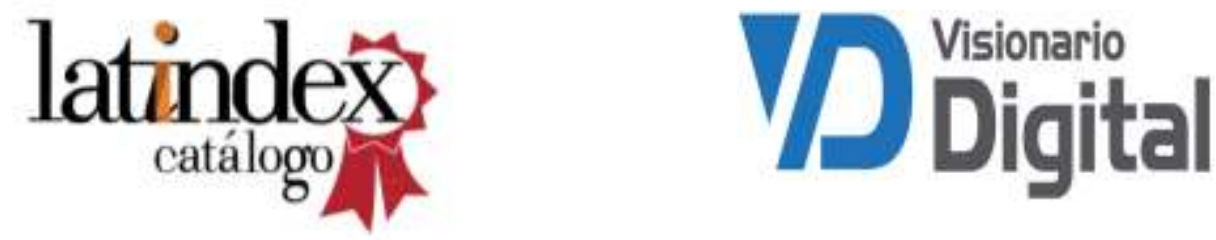